Pacific Journal of Mathematics

MORE ON A GENERALIZATION OF COMMUTATIVE AND . 


\title{
MORE ON A GENERALIZATION OF COMMUTATIVE AND ALTERNATIVE RINGS
}

\author{
Margaret Humm Kleinfeld
}

Let $R$ be a ring such that in every subring generated by two elements the following identities are satisfied:

$$
\begin{gathered}
\left(x, y^{2}, x\right)=y \circ(x, y, x) \\
(x, y, z)+(y, z, x)+(z, x, y)=0 \\
((x, y), x, x)=0,
\end{gathered}
$$

where $(a, b, c)=(a b) c-a(b c),(a, b)=a b-b a$, and $a \circ b=$ $a b+b a$. This condition is satisfied by any alternative ring and also by any commutative ring. Assume further that $R$ is a simple ring of characteristic not 2 or 3 and that $R$ has an idempotent $e$ such that $(e, e, R)=0=(R, e, e)$ while $(e, R) \neq 0$. It is proved in this paper that under these conditions $R$ must be alternative.

Main section. In [2] and [3], E. Kleinfeld has studied rings of characteristic not 2 or 3 which satisfy identities (1), (2), and (3). In [2] it is shown that if such a ring has no divisors of zero, it is either associative or commutative. In [3] it is shown that if such a ring $R$ is simple with idempotent $e$ such that $(e, e, R)=0=(R, e, e)$ and $(e, R) \neq 0$, then $R$ is associative. In [4] E. Kleinfeld considers rings of characteristic not 2 or 3 in which identities (1), (2), and (3) are assumed only locally, i.e., in subrings generated by two elements. Commutative rings satisfy this condition as do associative rings, but now in addition the new condition is satisfied by all alternative rings. In [4] it is proved that if such a ring has no divisors of zero, it must be either commutative or alternative. It remained to investigate the implications of the new local condition in the case of a simple ring $R$ with idempotent considered in [3]. This is done in the present paper. Henceforth we assume that $R$ is a simple ring of characteristic not 2 or 3 with idempotent $e$ satisfying $(e, e, R)=0=(R, e, e)$ and $(e, R) \neq 0$. We also assume that identities (1), (2), and (3) hold locally in $R$.

The full linearizations of identities (1) and (3) are respectively

$$
\begin{aligned}
A(w, y, z, x)= & (w, y \circ z, x)+(x, y \circ z, w)-y \circ(w, z, x) \\
& -z \circ(w, y, x)-y \circ(x, z, w)-z \circ(x, y, w)=0
\end{aligned}
$$


and

$$
\begin{aligned}
B(w, y, z, x)= & ((w, y), x, z)+((w, y), z, x)+((x, y), w, z) \\
& +((x, y), z, w)+((z, y), w, x)+((z, y), x, w)=0 .
\end{aligned}
$$

We will use the notation $J(x, y, z)=(x, y, z)+(z, x, y)+(y, z, x)$ to simplify some of the identities. Because (2) holds locally we have $J(x y, y, x)=0$. Linearizing $y$ gives

$$
C(x, y, z, x)=J(x y, z, x)+J(x z, y, x)=0,
$$

while linearizing $x$ in $J(x y, y, x)=0$, gives

$$
D(x, y, y, w)=J(x y, y, w)+J(w y, y, x)=0 .
$$

We also have $J(x y, x, y)=0$. Linearizing $y$ gives

$$
E(x, y, x, z)=J(x y, x, z)+J(x z, x, y)=0,
$$

while linearizing $x$ in $J(x y, x, y)=0$, gives

$$
F(x, y, w, y)=J(x y, w, y)+J(w y, x, y)=0 .
$$

Now $C(x, y, z, x)=0$ can be linearized to obtain

$$
\begin{aligned}
G(x, y, z, w)= & J(x y, z, w)+J(w y, z, x)+J(x z, y, w) \\
& +J(w z, y, x)=0 .
\end{aligned}
$$

Since we are assuming characteristics not 3 , the condition that (2) holds locally implies $(x, x, x)=0$, which can be linearized to give $J(x, y, z)+$ $J(x, z, y)=0$. This means the sum of the six possible associators with entries $x, y$, and $z$ is zero. This fact will be used freely and referred to simply as "third power associativity."

As in [3] we use the techniques employed by A. A. Albert in his paper on simple alternative rings [1], and we take the Peirce decomposition of $R$ into the direct sum, $R=R_{11} \oplus R_{10} \oplus R_{01} \oplus R_{00}$, where $e x_{i j}=i x_{i j}$ and $x_{i j} e=j x_{i j}$, for all $x_{i j} \in R_{i j}, i, j=0,1$. First we compute the multiplication table of the $R_{i j}$. Exactly as in [3] we can prove

$$
R_{10} R_{11}=0
$$

and

$$
R_{01} R_{00}=0
$$


The proof of $R_{00} R_{10}=0$ and $R_{10} R_{00} \subset R_{10}$ given in [3] has only one use of identity (2), and we can get the same thing by taking $0=C\left(e, y_{00}, x_{10}, e\right)=$ $J\left(e y_{00}, x_{10}, e\right)+J\left(e x_{10}, y_{00}, e\right)=J\left(x_{10}, y_{00}, e\right)$. Thus we have

$$
R_{00} R_{10}=0
$$

and

$$
R_{10} R_{00} \subset R_{10}
$$

When we reverse subscripts we can replace the use of (2) by

$$
0=E\left(e, y_{11}, e, x_{01}\right)=J\left(e y_{11}, e, x_{01}\right)+J\left(e x_{01}, e, y_{11}\right)=J\left(y_{11}, e, x_{01}\right) \text {, }
$$

and so we have

$$
R_{11} R_{01}=0
$$

and

$$
R_{01} R_{11} \subset R_{01}
$$

The proof in [3] of $R_{11} R_{10} \subset R_{10}$ also has only one use of identity (2) which can be replaced by using $F\left(y_{10}, e, x_{11}, e\right)=0$, and where subscripts are reversed, $D\left(x_{00}, e, e, y_{01}\right)=0$. Thus we have

$$
R_{11} R_{10} \subset R_{10}
$$

and

$$
R_{00} R_{01} \subset R_{01}
$$

Exactly as in [3] we can prove

$$
x_{10}^{2}=0=x_{10} y_{10}+y_{10} x_{10}
$$

and

$$
x_{01}^{2}=0=x_{01} y_{01}+y_{01} x_{01} \text {. }
$$

We cannot hope to prove $R_{10} R_{10}=0=R_{01} R_{01}$ as in [3], since this is true only for associative rings and not in general for alternative rings, and our hypotheses are satisfied by alternative rings. We can prove however that $R_{10} R_{10} \subset R_{01}$ and $R_{01} R_{01} \subset R_{10}$, which is what happens in 
alternative rings. These are the only two places where our multiplication inclusions will differ from those proved in [3]. We have $0=$ $B\left(e, x_{10}, y_{10}, e\right)=\left(\left(e, x_{10}\right), y_{10}, e\right)+\left(\left(e, x_{10}\right), e, y_{10}\right)+\left(\left(y_{10}, x_{10}\right), e, e\right)+$ $\left(\left(y_{10}, x_{10}\right), e, e\right)+\left(\left(e, x_{10}\right), e, y_{10}\right)+\left(\left(e, x_{10}\right), y_{10}, e\right)=$ $2\left(x_{10}, y_{10}, e\right)+2\left(x_{10}, e, y_{10}\right)$. Thus we have $\left(x_{10}, y_{10}, e\right)+\left(x_{10}, e, y_{10}\right)=$ 0 . Expansion gives $x_{10} y_{10} \cdot e-x_{10} y_{10}=0$ so that

$$
x_{10} y_{10} \cdot e=x_{10} y_{10} .
$$

Also we have $0=A\left(x_{10}, y_{10}, e, e\right)=\left(x_{10}, y_{10} \circ e, e\right)+\left(e, y_{10} \circ e, x_{10}\right)-$ $y_{10} \circ\left(x_{10}, e, e\right)-e \circ\left(x_{10}, y_{10}, e\right)-y_{10} \circ\left(e, e, x_{10}\right)-e \circ\left(e, y_{10}, x_{10}\right)=$ $\left(x_{10}, y_{10}, e\right)+\left(e, y_{10}, x_{10}\right)-e \circ\left(x_{10}, y_{10}, e\right)-e \circ\left(e, y_{10}, x_{10}\right)$. Thus we have $\left(x_{10}, y_{10}, e\right)+\left(e, y_{10}, x_{10}\right)=e \circ\left[\left(x_{10}, y_{10}, e\right)+\right.$ $\left.\left(e, y_{10}, x_{10}\right)\right]$. However, expansion gives $\left(x_{10}, y_{10}, e\right)+\left(e, y_{10}, x_{10}\right)=$ $x_{10} y_{10} \cdot e+y_{10} x_{10}-e \cdot y_{10} x_{10}=e \cdot x_{10} y_{10}$, because of (12) and (14). Substituting this in the previous equation we get $e \cdot x_{10} y_{10}=$ $e \circ\left(e \cdot x_{10} y_{10}\right)$. Since $(e, e, R)=(R, e, e)=0$, which also implies $(e, R, e)=0$, the previous equation reduces to $e \cdot x_{10} y_{10} \cdot e=0$, which in view of (14) gives

$$
e \cdot x_{10} y_{10}=0 \text {. }
$$

Combining (14) and (15), we see that we have proved

$$
R_{10} R_{10} \subset R_{01}
$$

The same substitutions with subscripts reversed yield a proof of

$$
R_{01} R_{01} \subset R_{10}
$$

The proof of $R_{10} R_{01} \subset R_{11}$ in [3] has only one use of (2) which can be replaced by $C\left(e, x_{10}, y_{01}, e\right)=0$, and with subscripts reversed by $F\left(x_{01}, e, y_{10}, e\right)=0$. Thus we have

$$
R_{10} R_{01} \subset R_{11}
$$

and

$$
R_{01} R_{10} \subset R_{00}
$$

We now wish to consider products of the type $R_{00} R_{11}$ and $R_{11} R_{00}$. We have $0=F\left(y_{11}, e, x_{00}, e\right)=J\left(y_{11} e, x_{00}, e\right)+J\left(x_{00} e, y_{11}, e\right)=J\left(y_{11}, x_{00}, e\right)$, and $0=E\left(e, y_{11}, e, x_{00}\right)=J\left(e y_{11}, e, x_{00}\right)+J\left(e x_{00}, e, y_{11}\right)=$ 
$J\left(y_{11}, e, x_{00}\right)$. Thus we have $J\left(y_{11}, x_{00}, e\right)=0$ and $J\left(y_{11}, e, x_{00}\right)=0$. Using these two equations, we can proceed exactly as in [3] to prove

$$
x_{00} y_{11}=y_{11} x_{00} \in R_{00}+R_{11} \text {. }
$$

We now consider products of the form $R_{11} R_{11}$. From third power associativity we have $\left(x_{11}, y_{11}, e\right)+\left(e, x_{11}, y_{11}\right)+\left(y_{11}, e, x_{11}\right)+\left(y_{11}, x_{11}, e\right)$ $+\left(e, y_{11}, x_{11}\right)+\left(x_{11}, e, y_{11}\right)=0$. Expanding and simplifying we get $\left(x_{11} y_{11}+y_{11} x_{11}\right) e-e\left(x_{11} y_{11}+y_{11} x_{11}\right)=0$, which can be rewritten as $\left(x_{11} \circ y_{11}, e\right)=0$. This implies

$$
x_{11}^{\circ} y_{11} \in R_{11}+R_{00} .
$$

Now $A\left(x_{11}, y_{11}, e, e\right)=0$ gives $2\left(x_{11}, y_{11}, e\right)+2\left(e, y_{11}, x_{11}\right)=$ $e \circ\left(x_{11}, y_{11}, e\right)+e \circ\left(e, y_{11}, x_{11}\right)$. Expanding and simplifying gives $2\left(x_{11} y_{11} \cdot e-x_{11} y_{11}+y_{11} x_{11}-e \cdot y_{11} x_{11}\right)=e \cdot x_{11} y_{11} \cdot e-e \cdot x_{11} y_{11}+$ $y_{11} x_{11} \cdot e-e \cdot y_{11} x_{11} \cdot e$. Now if we let $x_{11} y_{11}=a_{11}+a_{10}+a_{01}+a_{00}$, then because of (21) we have $y_{11} x_{11}=b_{11}-a_{10}-a_{01}+b_{00}$. Substituting these in the preceding equation yields $-2 a_{00}-a_{10}-a_{01}+2 b_{00}=0$. By the directness of the Peirce decomposition this gives $a_{10}=a_{01}=0$ and $a_{00}=b_{00}$. Thus we have

$$
R_{11} R_{11} \subset R_{11}+R_{00}
$$

and

$$
\left(x_{11} y_{11}\right)_{00}=\left(y_{11} x_{11}\right)_{00} \text {. }
$$

Reversing subscripts yields a proof that

$$
R_{00} R_{00} \subset R_{11}+R_{00}
$$

and

$$
\left(x_{00} y_{00}\right)_{11}=\left(y_{00} x_{00}\right)_{11} \text {. }
$$

From $B\left(e, x_{10}, y_{11}, z_{00}\right)=0$ one can prove $x_{10} \cdot y_{11} z_{00}=0=x_{10} \cdot z_{00} y_{11}$, exactly as in [3]. Using this, (4), and (7) we have $\left(x_{10}, y_{11}, z_{00}\right)=0=$ $\left(x_{10}, z_{00}, y_{11}\right)$. Also, using (4) and (6), we see that $\left(z_{00}, x_{10}, y_{11}\right)=0$. Now from $A\left(z_{00}, y_{11}, e, x_{10}\right)=0$ we get $2\left(z_{00}, y_{11}, x_{10}\right)+2\left(x_{10}, y_{11}, z_{00}\right)=$ $e \circ\left(z_{00}, y_{11}, x_{10}\right)+e \circ\left(x_{10}, y_{11}, z_{00}\right)+y_{11} \circ\left(z_{00}, e, x_{10}\right)+y_{11} \circ\left(x_{10}, e, z_{00}\right)$. We already know $\left(x_{10}, y_{11}, z_{00}\right)=0$ and using (6) we see that $\left(z_{00}, e, x_{10}\right)=0$ while clearly $\left(x_{10}, e, z_{00}\right)=0$. We are left with 


$$
2\left(z_{00}, y_{11}, x_{10}\right)=e \circ\left(z_{00}, y_{11}, x_{10}\right)
$$

On the other hand, expanding and using (6), (10), and (20) we see that $\left(z_{00}, y_{11}, x_{10}\right) \in R_{10}$, so that we have

$$
e \circ\left(z_{00}, y_{11}, x_{10}\right)=\left(z_{00}, y_{11}, x_{10}\right) \text {. }
$$

Combining (26) and (27) we conclude that $\left(z_{00}, y_{11}, x_{10}\right)=0$. Now expanding and using (6), (10), and (20) gives $\left(y_{11}, z_{00}, x_{10}\right)=y_{11} z_{00} \cdot x_{10}=$ $z_{00} y_{11} \cdot x_{10}=\left(z_{00}, y_{11}, x_{10}\right)=0$. We have now shown that five of the six possible associators involving $y_{11}, z_{00}$ and $x_{10}$ are zero. By third power associativity the remaining one, $\left(y_{11}, x_{10}, z_{00}\right)$, must be zero also. We have proved

$$
\begin{aligned}
\left(x_{10}, y_{11}, z_{00}\right) & =\left(y_{11}, z_{00}, x_{10}\right)=\left(z_{00}, x_{10}, y_{11}\right) \\
& =\left(z_{00}, y_{11}, x_{10}\right)=\left(y_{11}, x_{10}, z_{00}\right)=\left(x_{10}, z_{00}, y_{11}\right)=0 .
\end{aligned}
$$

The same substitutions with subscripts reversed yield a proof of

$$
\begin{aligned}
\left(x_{01}, y_{11}, z_{00}\right) & =\left(y_{11}, z_{00}, x_{01}\right)=\left(z_{00}, x_{01}, y_{11}\right) \\
& =\left(z_{00}, y_{11}, x_{01}\right)=\left(y_{11}, x_{01}, z_{00}\right)=\left(x_{01}, z_{00}, y_{11}\right)=0 .
\end{aligned}
$$

As in [3] we let $A=\left\{a_{11}+a_{00} / a_{11} R_{10}=R_{01} a_{11}=0=a_{00} R_{01}=\right.$ $\left.R_{10} a_{00}\right\}$. We can prove exactly as in [3] that

$$
R_{11} R_{00} \subset A \quad \text { and } \quad R_{00} R_{11} \subset A .
$$

We now wish to show that all associators with two elements from $R_{i i}$ and one element from $R_{i j}$ vanish. From $B\left(x_{10}, e, y_{11}, z_{11}\right)=0$ we get $0=\left(x_{10}, y_{11}, z_{11}\right)+\left(x_{10}, z_{11}, y_{11}\right)=-x_{10}\left(y_{11} z_{11}+z_{11} y_{11}\right)$. Using (22) and (23), let $y_{11} z_{11}=a_{11}+a_{00}$ and $z_{11} y_{11}=b_{11}+a_{00}$. Then we have $0=$ $x_{10}\left(y_{11} z_{11}+z_{11} y_{11}\right)=x_{10}\left(a_{11}+b_{11}+2 a_{00}\right)=2 x_{10} a_{00}$. Thus we have $x_{10} a_{00}=0$. But this implies that $\left(x_{10}, y_{11}, z_{11}\right)=-x_{10}\left(a_{11}+a_{00}\right)=0$ and so also $\left(x_{10}, z_{11}, y_{11}\right)=0$. Now from $A\left(x_{10}, e, y_{11}, z_{11}\right)=0$ we get $2\left(z_{11}, y_{11}, x_{10}\right)=e \circ\left(z_{11}, y_{11}, x_{10}\right)$ but since $\left(z_{11}, y_{11}, x_{10}\right) \in R_{10}$, this implies $\left(z_{11}, y_{11}, x_{10}\right)=0$. The same argument with $z_{11}$ and $y_{11}$ interchanged proves that $\left(y_{11}, z_{11}, x_{10}\right)=0$ and because of (4) and (10) we have directly that $\left(z_{11}, x_{10}, y_{11}\right)=0=\left(y_{11}, x_{10}, z_{11}\right)$. Thus we have

$$
\left(y_{11}, z_{11}, x_{10}\right)=\left(y_{11}, x_{10}, z_{11}\right)=\left(x_{10}, y_{11}, z_{11}\right)=0 \text {. }
$$

The same substitutions with subscripts reversed yield a proof that

$$
\left(x_{01}, y_{00}, z_{00}\right)=\left(y_{00}, x_{01}, z_{00}\right)=\left(y_{00}, z_{00}, x_{01}\right)=0 \text {. }
$$


We now show that all associators with two entries from $R_{i i}$ and one entry from $R_{j i}$ vanish. From $B\left(x_{01}, e, y_{11}, z_{11}\right)=0$ we get $\left(x_{01}, y_{11}, z_{11}\right)+$ $\left(x_{01}, z_{11}, y_{11}\right)=0$. Because of (8) and (9) we have directly that $\left(y_{11}, x_{01}, z_{11}\right)=0=\left(z_{11}, x_{01}, y_{11}\right)$. It follows by third power associativity that $\left(y_{11}, z_{11}, x_{01}\right)+\left(z_{11}, y_{11}, x_{01}\right)=0$. Expanding gives $\left(y_{11} z_{11}+z_{11} y_{11}\right) x_{01}=0$. Using (22) and (23), let $y_{11} z_{11}=a_{11}+a_{00}$ and $z_{11} y_{11}=b_{11}+a_{00}$. Substituting this in the preceding equation gives $\left(a_{11}+b_{11}+2 a_{00}\right) x_{01}=0$, and hence $a_{00} x_{01}=0$. We now have $\left(y_{11}, z_{11}, x_{01}\right)=0=\left(z_{11}, y_{11}, x_{01}\right)$. Now $A\left(x_{01}, e, y_{11}, z_{11}\right)=0 \quad$ gives $2\left(x_{01}, y_{11}, z_{11}\right)=e \circ\left(x_{01}, y_{11}, z_{11}\right)$, but $\left(x_{01}, y_{11}, z_{11}\right) \in R_{01}$ because of (5), (9), and (22). Thus $\left(x_{01}, y_{11}, z_{11}\right)=0$. We have now shown

$$
\left(x_{01}, y_{11}, z_{11}\right)=\left(y_{11}, x_{01}, z_{11}\right)=\left(y_{11}, z_{11}, x_{01}\right)=0 \text {. }
$$

Reversing subscripts yields a proof of

$$
\left(x_{10}, y_{00}, z_{00}\right)=\left(y_{00}, x_{10}, z_{00}\right)=\left(y_{00}, z_{00}, x_{10}\right)=0 \text {. }
$$

We can now prove exactly as in [3] that $A$ is an ideal of $R$ and that since $R$ is simple and $(e, R) \neq 0$, we must have $A=0$. Since $R_{11} R_{00} \subset A$ and $R_{00} R_{11} \subset A$ by (30), we have

$$
R_{11} R_{00}=R_{00} R_{11}=0
$$

Also using (31)-(34) we can prove easily that if $y_{11} z_{11}=a_{11}+a_{00}$, then $a_{00} \in A$ and if $y_{00} z_{00}=c_{11}+c_{00}$ then $c_{11} \in A$, in fact our proofs of equations (31)-(34) contain this information. Thus we have

$$
R_{11} R_{11} \subset R_{11}
$$

and

$$
R_{00} R_{00} \subset R_{00}
$$

We now have the same multiplication table as in an alternative ring, i.e., $R_{i j} R_{k l} \subset \delta_{j k} R_{i l}$ except for the case $R_{i j} R_{i j}$ where we have $R_{i j} R_{i j} \subset$ $R_{j i}$. We will now show that $R$ must be alternative by considering all possible associators $\left(R_{i j}, R_{k l}, R_{m n}\right)$ and showing that they obey the alternative law. An associator $(x, y, z)$ obeys the alternative law if $(x \sigma, y \sigma, z \sigma)=\operatorname{sgn} \sigma(x, y, z)$ for all six permutations $\sigma$ of $x, y$, and $z$ within the associator. Using (31), (33), (35), and (36) it is obvious that all associators with exactly two entries from $R_{i i}$ vanish, $i=0,1$. Thus assuming $i \neq j$ we have 


$$
\left(R_{i i} \sigma, R_{i i} \sigma, R_{i j} \sigma\right)=\left(R_{i i} \sigma, R_{i i} \sigma, R_{i i} \sigma\right)=\left(R_{i i} \sigma, R_{i i} \sigma, R_{i j} \sigma\right)=0 \text {. }
$$

In the case where exactly one entry is from $R_{i i}$, we see that if one or both of the remaining entries are from $R_{i j}$, the associator vanishes by either (28), (29), or (38). There are three other possibilities for the remaining two entries, both from $R_{i j}$, both from $R_{j i}$, or one from $R_{i j}$ and the other from $R_{j i}$. We consider the last of these first. We have immediately on expansion that $\left(x_{11}, y_{01}, z_{10}\right)=0, \quad\left(y_{01}, z_{10}, x_{11}\right)=0$ and $\left(z_{10}, x_{11}, y_{01}\right)=$ 0 . Now from $B\left(y_{01}, e, x_{11}, z_{10}\right)=0$ we get $\left(y_{01}, x_{11}, z_{10}\right)+\left(y_{01}, z_{10}, x_{11}\right)-$ $\left(z_{10}, y_{01}, x_{11}\right)-\left(z_{10}, x_{11}, y_{01}\right)=0$. The second and fourth terms vanish leaving $\quad\left(y_{01}, x_{11}, z_{10}\right)=\left(z_{10}, y_{01}, x_{11}\right)$, but $\quad\left(y_{01}, x_{11}, z_{10}\right) \in R_{00} \quad$ while $\left(z_{10}, y_{01}, x_{11}\right) \in R_{11}$, hence we must have $\left(y_{01}, x_{11}, z_{10}\right)=0=\left(z_{10}, y_{01}, x_{11}\right)$. It follows from third power associativity that $\left(x_{11}, z_{10}, y_{01}\right)=0$ also. Since we could repeat this with subscripts reversed, we have

$$
\left(R_{i i} \sigma, R_{i j} \sigma, R_{i i} \sigma\right)=0 .
$$

Now consider associators of the form $\left(R_{11} \sigma, R_{10} \sigma, R_{10} \sigma\right)$. Using (4), (10), and (12) we see that $\left(x_{11}, y_{10}, z_{10}\right)=x_{11} y_{10} \cdot z_{10}=-z_{10} \cdot x_{11} y_{10}=$ $\left(z_{10}, x_{11}, y_{10}\right)$, so we have

$$
\left(x_{11}, y_{10}, z_{10}\right)=\left(z_{10}, x_{11}, y_{10}\right)
$$

Since $y_{10}$ and $z_{10}$ are interchangeable, it follows from (40) that

$$
\left(x_{11}, z_{10}, y_{10}\right)=\left(y_{10}, x_{11}, z_{10}\right) \text {. }
$$

We also have $\left(y_{10}, z_{10}, x_{11}\right)=y_{10} z_{10} \cdot x_{11}=-z_{10} y_{10} \cdot x_{11}=-\left(z_{10}, y_{10}, x_{11}\right)$. Thus we have

$$
\left(y_{10}, z_{10}, x_{11}\right)=-\left(z_{10}, y_{10}, x_{11}\right) \text {. }
$$

Using third power associativity we have $\left(x_{11}, y_{10}, z_{10}\right)+\left(z_{10}, x_{11}, y_{10}\right)+$ $\left(y_{10}, z_{10}, x_{11}\right)+\left(x_{11}, z_{10}, y_{10}\right)+\left(y_{10}, x_{11}, z_{10}\right)+\left(z_{10}, y_{10}, x_{11}\right)=$ 0 . Substituting in this equation (40), (41), and (42), we find this reduces to $2\left(x_{11}, y_{10}, z_{10}\right)+2\left(x_{11}, z_{10}, y_{10}\right)=0$, so we have

$$
\left(x_{11}, y_{10}, z_{10}\right)=-\left(x_{11}, z_{10}, y_{10}\right) \text {. }
$$

Combining (40), (43), and (41) we see that four of the associators are related by 


$$
\begin{aligned}
\left(z_{10}, x_{11}, y_{10}\right) & =\left(x_{11}, y_{10}, z_{10}\right)=-\left(x_{11}, z_{10}, y_{10}\right) \\
& =-\left(y_{10}, x_{11}, z_{10}\right)
\end{aligned}
$$

and the remaining two are related by (42). We need only link one of the first four to one of the second two in the proper way to have the full alternative relation. For this we use $G\left(x_{11}, e, z_{10}, y_{10}\right)=0$ to get $\left(x_{11}, z_{10}, y_{10}\right)+\left(y_{10}, x_{11}, z_{10}\right)+\left(z_{10}, y_{10}, x_{11}\right)+\left(x_{11} z_{10}, e, y_{10}\right)+\left(y_{10}, x_{11} z_{10}, e\right)$ $+\left(e, y_{10}, x_{11} z_{10}\right)+\left(y_{10} z_{10}, e, x_{11}\right)+\left(x_{11}, y_{10} z_{10}, e\right)+\left(e, x_{11}, y_{10} z_{10}\right)=$ 0. Expanding the associators and using the multiplicative relations, we see that the last three associators vanish. Using this and expanding the associators containing the product $x_{11} z_{10}$ gives $\left(x_{11}, z_{10}, y_{10}\right)+\left(y_{10}, x_{11}, z_{10}\right)$ $+\left(z_{10}, y_{10}, x_{11}\right)-x_{11} z_{10} \cdot y_{10}+y_{10} \cdot x_{11} z_{10}+y_{10} \cdot x_{11} z_{10}=0$. Using (4), (10), and (12) we see that $-x_{11} z_{10} \cdot y_{10}=y_{10} \cdot x_{11} z_{10}=$ $-\left(y_{10}, x_{11}, z_{10}\right)$. Substituting this in the preceding equation gives $\left(x_{11}, z_{10}, y_{10}\right)+\left(y_{10}, x_{11}, z_{10}\right)+\left(z_{10}, y_{10}, x_{11}\right)-3\left(y_{10}, x_{11}, z_{10}\right)=0$. By (41) and (44) this yields $-\left(x_{11}, z_{10}, y_{10}\right)+\left(z_{10}, y_{10}, x_{11}\right)=0$. We have now shown

$$
\left(R_{i i} \sigma, R_{i j} \sigma, R_{i j} \sigma\right)=\operatorname{sgn} \sigma\left(R_{i i}, R_{i j}, R_{i j}\right)
$$

We now consider the possibility of one entry in $R_{11}$ and two entries in $\boldsymbol{R}_{01}$. This works very similarly to the preceding argument. By expanding and using (8), (9), and (13), we quickly get $\left(z_{01}, y_{01}, x_{11}\right)=$ $\left(y_{01}, x_{11}, z_{01}\right), \quad\left(y_{01}, z_{01}, x_{11}\right)=\left(z_{01}, x_{11}, y_{01}\right) \quad$ and $\quad\left(x_{11}, y_{01}, z_{01}\right)=$ $-\left(x_{11}, z_{01}, y_{01}\right)$. Substituting this in third power associativity one gets $\left(z_{01}, x_{11}, y_{01}\right)=-\left(y_{01}, x_{11}, z_{01}\right)$. Thus we have

$$
\begin{aligned}
\left(z_{01}, y_{01}, x_{11}\right) & =\left(y_{01}, x_{11}, z_{01}\right)=-\left(z_{01}, x_{11}, y_{01}\right) \\
& =-\left(y_{01}, z_{01}, x_{11}\right)
\end{aligned}
$$

and

$$
\left(x_{11}, y_{01}, z_{01}\right)=-\left(x_{11}, z_{01}, y_{01}\right) \text {. }
$$

The final link is provided by $G\left(e, x_{11}, z_{01}, y_{01}\right)=0$, which gives $\left(x_{11}, z_{01}, y_{01}\right)$ $+\left(y_{01}, x_{11}, z_{01}\right)+\left(z_{01}, y_{01}, x_{11}\right)+\left(y_{01} x_{11}, z_{01}, e\right)+\left(e, y_{01} x_{11}, z_{01}\right)+$ $\left(z_{01}, e, y_{01} x_{11}\right)+\left(y_{01} z_{01}, x_{11}, e\right)+\left(e, y_{01} z_{01}, x_{11}\right)+\left(x_{11}, e, y_{01} z_{01}\right)=0$. The last three terms can be shown to vanish by expansion. Expanding the associators involving $y_{01} x_{11}$, the preceding equation becomes $\left(x_{11}, z_{01}, y_{01}\right)$ $+\left(y_{01}, x_{11}, z_{01}\right)+\left(z_{01}, y_{01}, x_{11}\right)-y_{01} x_{11} \cdot z_{01}-y_{01} x_{11} \cdot z_{01}+z_{01} \cdot y_{01} x_{11}=$ 0 . Using (8) and (13) this gives $\left(x_{11}, z_{01}, y_{01}\right)+\left(y_{01}, x_{11}, z_{01}\right)+$ $\left(z_{01}, y_{01}, x_{11}\right)-3\left(y_{01}, x_{11}, z_{01}\right)=0$. Using (46) this reduces to $\left(x_{11}, z_{01}, y_{01}\right)-$ 
$\left(y_{01}, x_{11}, z_{01}\right)=0$. Since subscripts can be reversed in this proof. we have proved

$$
\left(R_{i i} \sigma, R_{i i} \sigma, R_{i i} \sigma\right)=\operatorname{sgn} \sigma\left(R_{i i}, R_{i i}, R_{j i}\right)
$$

This completes the case where exactly one entry comes from $R_{i i}$. Next we consider the case where all entries are from $R_{10}$. From $A\left(y_{10}, e, z_{10}, x_{10}\right)=0$ we get $\left(y_{10}, z_{10}, x_{10}\right)+\left(x_{10}, z_{10}, y_{10}\right)=e \circ\left(y_{10}, z_{10}, x_{10}\right)$ $+z_{10} \circ\left(y_{10}, e, x_{10}\right)+e \circ\left(x_{10}, z_{10}, y_{10}\right)+z_{10} \circ\left(x_{10}, e, y_{10}\right)$. Expanding we see that $z_{10}{ }^{\circ}\left(y_{10}, e, x_{10}\right)+z_{10} \circ\left(x_{10}, e, y_{10}\right)=z_{10} \circ\left(-y_{10} x_{10}-x_{10} y_{10}\right)=0$ because of (12). Thus we are left with

$$
\begin{aligned}
\left(y_{10}, z_{10}, x_{10}\right)+\left(x_{10}, z_{10}, y_{10}\right) & \\
= & e \circ\left[\left(y_{10}, z_{10}, x_{10}\right)+\left(x_{10}, z_{10}, y_{10}\right)\right] .
\end{aligned}
$$

Expanding will show that $\left(R_{10}, R_{10}, R_{10}\right) \subset R_{11}+R_{00}$. Let $\left(y_{10}, z_{10}, x_{10}\right)+$ $\left(x_{10}, z_{10}, y_{10}\right)=a_{11}+a_{00}$. Then $e \circ\left[\left(y_{10}, z_{10}, x_{10}\right)+\left(x_{10}, z_{10}, y_{10}\right)\right]=$ $2 a_{11}$. Comparison with (49) yields $2 a_{11}=a_{11}+a_{00}$ which implies $a_{11}=$ $0=a_{00}$, so that we have

$$
\left(y_{10}, z_{10}, x_{10}\right)=-\left(x_{10}, z_{10}, y_{10}\right)
$$

From $B\left(x_{10}, y_{10}, e, z_{10}\right)=0$, we get $\left(\left(x_{10}, y_{10}\right), e, z_{10}\right)+\left(\left(x_{10}, y_{10}\right), z_{10}, e\right)+$ $\left(\left(e, y_{10}\right), x_{10}, z_{10}\right)+\left(\left(e, y_{10}\right), z_{10}, x_{10}\right)+\left(\left(z_{10}, y_{10}\right), x_{10}, e\right)+\left(\left(z_{10}, y_{10}\right), e, x_{10}\right)=$ 0 . The first two terms and the last two terms vanish because of (39). We are left with $\left(y_{10}, x_{10}, z_{10}\right)+\left(y_{10}, z_{10}, x_{10}\right)=0$ which gives

$$
\left(y_{10}, x_{10}, z_{10}\right)=-\left(y_{10}, z_{10}, x_{10}\right)
$$

Since all entries are from $R_{10}$ and since any two transpositions generate the full symmetric group on three elements, (50) and (51) are sufficient to imply the full alternative law for associators of this type. Since the same argument works with subscripts reversed, we have proved

$$
\left(R_{i j} \sigma, R_{i j} \sigma, R_{i j}\right)=\operatorname{sgn} \sigma\left(R_{i j}, R_{i j}, R_{i j}\right) .
$$

Now we consider the case of one entry from $R_{01}$ and two entries from $R_{10}$. Using (12), (13), and various multiplicative relation we have $\left(x_{01}, y_{10}, z_{10}\right)=\left(y_{10}, z_{10}, x_{01}\right), \quad\left(x_{01}, y_{10}, z_{10}\right)=-\left(x_{01}, z_{10}, y_{10}\right)$, and $\left(x_{01}, z_{10}, y_{10}\right)=\left(z_{10}, y_{10}, x_{01}\right)$. Thus we have four of the associators properly related by

$$
\begin{aligned}
\left(x_{01}, y_{10}, z_{10}\right) & =\left(y_{10}, z_{10}, x_{01}\right)=-\left(x_{01}, z_{10}, y_{10}\right) \\
& =-\left(z_{10}, y_{10}, x_{01}\right) .
\end{aligned}
$$


From third power associativity we now get

$$
\left(y_{10}, x_{01}, z_{10}\right)=-\left(z_{10}, x_{01}, y_{10}\right)
$$

The necessary link is provided by $G\left(x_{01}, e, z_{10}, y_{10}\right)=0=\left(x_{01}, z_{10}, y_{10}\right)+$ $\left(y_{10}, x_{01}, z_{10}\right)+\left(z_{10}, y_{10}, x_{01}\right)+\left(x_{01} z_{10}, e, y_{10}\right)+\left(y_{10}, x_{01} z_{10}, e\right)+$ $\left(e, y_{10}, x_{01} z_{10}\right)+\left(y_{10} z_{10}, e, x_{01}\right)+\left(x_{01}, y_{10} z_{10}, e\right)+\left(e, x_{01}, y_{10} z_{10}\right)$. In this equation all the associators having entry $x_{01} z_{10}$ vanish because of (19) and (28). We are left with $\left(x_{01}, z_{10}, y_{10}\right)+\left(y_{10}, x_{01}, z_{10}\right)+\left(z_{10}, y_{10}, x_{01}\right)+$ $y_{10} z_{10} \cdot x_{01}-x_{01} \cdot y_{10} z_{10}-x_{01} \cdot y_{10} z_{10}=0$. This gives $\left(x_{01}, z_{10}, y_{10}\right)+$ $\left(y_{10}, x_{01}, z_{10}\right)+\left(z_{10}, y_{10}, x_{01}\right)+3\left(x_{01}, y_{10}, z_{10}\right)=0$. Using (53) this reduces to $\left(x_{01}, y_{10}, z_{10}\right)+\left(y_{10}, x_{01}, z_{10}\right)=0$, which completes the case. Since we can reverse subscripts in this proof we have proved

$$
\left(R_{i j} \sigma, R_{i j} \sigma, R_{j i} \sigma\right)=\operatorname{sgn} \sigma\left(R_{i j}, R_{i j}, R_{j i}\right) .
$$

We are now left with only the cases where all three entries come from the same $R_{i i}$. We will show that $R_{11}$ and $R_{00}$ are associative subrings of $R$. As in [1], it follows easily from the multiplicative relations of the $R_{i j}$ that $B=R_{10} R_{01}+R_{10}+R_{01}+R_{01} R_{10}$ is an ideal of $R$. Since we are assuming $(e, R) \neq 0$, we have $R_{10}+R_{01} \neq 0$, so that $B \neq 0$. Hence by the simplicity of $R$ we have $B=R$. This implies that $R_{11}=R_{10} R_{01}$ and $R_{00}=R_{01} R_{10}$. Now consider the associator $\left(x_{11}, y_{11}, z_{11}\right)$. Since $R_{11}=$ $R_{10} R_{01}$, we can write $\left(x_{11}, y_{11}, z_{11}\right)$ as a sum of associators of the form $\left(a_{10} b_{01}, y_{11}, z_{11}\right)$. In an arbitrary ring we have the Teichmüller identity $(w x, y, z)-(w, x y, z)+(w, x, y z)=w(x, y, z)+$ $(w, x, y) z$. Using this gives $\left(a_{10} b_{01}, y_{11}, z_{11}\right)-\left(a_{10}, b_{01} y_{11}, z_{11}\right)+$ $\left(a_{10}, b_{01}, y_{11} z_{11}\right)=a_{10}\left(b_{01}, y_{11}, z_{11}\right)+\left(a_{10}, b_{01}, y_{11}\right) z_{11}$. However, by (39) associators of the type $\left(R_{10}, R_{01}, R_{11}\right)$ vanish and by (33) those of the type $\left(R_{01}, R_{11}, R_{11}\right)$ vanish also. We are left with $\left(a_{10} b_{01}, y_{11}, z_{11}\right)=0$ and hence $\left(x_{11}, y_{11}, z_{11}\right)=0$ so that $R_{11}$ is associative. A similar argument proves that $R_{00}$ is associative. We have proved the following:

THEOREM. If $R$ is a simple ring of characteristic not 2 or 3 , which has an idempotent $e$ such that $(e, e, R)=0=(R, e, e)$ and $(e, R) \neq 0$, and if every subring of $R$ generated by two elements satisfies (1), (2), and (3), then $R$ is alternative and hence either associative or a Cayley Dickson algebra [1].

\section{REFERENCES}

1. A. A. Albert, On simple alternative rings, Canad. J. Math., 4 (1952), 129-135.

2. Erwin Kleinfeld, A generalization of commutative and associative rings, Pacific J. Math., 38 (1971), 95-101. 
3. More on a generalization of commutative and associative rings, Scripta Mathematica, Vol 'XXIX. No. 3-4, pp. 351-357.

4. - A generalization of commutative and alternative rings IV, Proc. Amer. Math. Soc., 46 (1974), 21-230.

Received August 7, 1973.

UNIVERSITY OF IOWA 



\section{Pacific Journal of Mathematics}

\section{Vol. 56, No. $1 \quad$ November, 1975}

Shimshon A. Amitsur, Central embeddings in semi-simple rings .......... 1

David Marion Arnold and Charles Estep Murley, Abelian groups, A, such

that $\operatorname{Hom}(A,---)$ preserves direct sums of copies of $A \ldots \ldots \ldots .$.

Martin Bartelt, An integral representation for strictly continuous linear

operators ................................... 21

Richard G. Burton, Fractional elements in multiplicative lattices......... 35

James Alan Cochran, Growth estimates for the singular values of

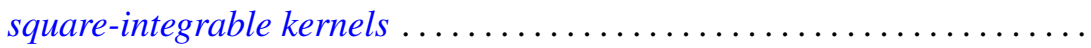

C. Martin Edwards and Peter John Stacey, On group algebras of central

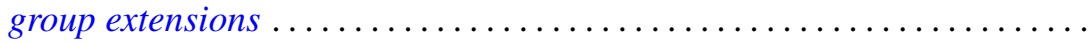

Peter Fletcher and Pei Liu, Topologies compatible with homeomorphism

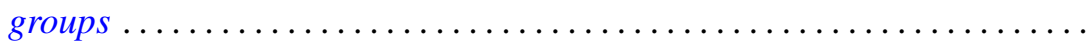

George Gasper, Jr., Products of terminating ${ }_{3} F_{2}(1)$ series ............ 87

Leon Gerber, The orthocentric simplex as an extreme simplex ............

Burrell Washington Helton, A product integral solution of a Riccati

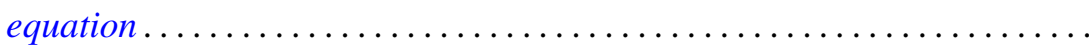

Melvyn W. Jeter, On the extremal elements of the convex cone of

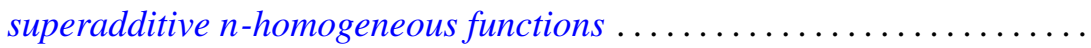

R. H. Johnson, Simple separable graphs .

Margaret Humm Kleinfeld, More on a generalization of commutative and

alternative rings. . .

A. Y. W. Lau, The boundary of a semilattice on an $n$-cell.

Robert F. Lax, The local rigidity of the moduli scheme for curves ...

Glenn Richard Luecke, A note on quasidiagonal and quasitriangular

operators .

Paul Milnes, On the extension of continuous and almost periodic functions

Hidegoro Nakano and Kazumi Nakano, Connector theory.

James Michael Osterburg, Completely outer Galois theory of perfect rings ..................................

Lavon Barry Page, Compact Hankel operators and the F. and M. Riesz

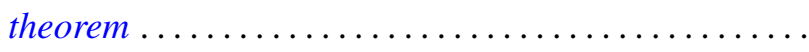

Joseph E. Quinn, Intermediate Riesz spaces..................... 225

Shlomo Vinner, Model-completeness in a first order language with a generalized quantifier.

Jorge Viola-Prioli, On absolutely torsion-free rings ..........

Philip William Walker, A note on differential equations with all solutions of integrable-square............................

Stephen Jeffrey Willson, Equivariant maps between representation 